werden, weil ihre Darstellung in genügender Quantităt zu zeitraubend gewesen wäre.

Um das Phenylhydrazin in Sulfonsäuren äberzuführen, hat Hr. Biel mehrere Versuche angestellt. Beim Erhitzen von schwefelsaurem Phenylhydrazin mit Schwefelsäurechlorhydrin auf $160^{\circ}$ entsteht die Phenylhydrazinsulfonsäure, $\mathrm{C}_{6} \mathrm{H}_{4} \cdot \mathrm{N}_{2} \mathrm{H}_{3} . \mathrm{SO}_{3} \mathrm{H}$. Die Lösung des Retorteninhalts in heissem Wasser setzt beim Erkalten Krystalle der Säure ab, die noch mit Thierkohle entfärbt werden. Dieselbe Säure entsteht beim Erhitzen des äthylschwefelsauren Phenylhydrazins auf $160^{\circ}$, wobei Weingeist überdestillirt. Sie krystallisirt in schwach gelb gefärbten, perlmutterglänzenden Blăttchen und zeigt die charakteristischen Eigenschaften der Hydrazinverbindungen.

Die Analyse lieferte:

$\begin{array}{lcc} & \text { Berechnet } & \text { Gefunden } \\ \mathrm{N} & 14.9 & 15.1 \mathrm{pCt} . \\ \mathrm{S} & 17.0 & 17.2\end{array}$

Das Baryumsalz ist sehr leicht löslich in Wasser und wird durch Alkohol als Krystallpulver gefällt. Beim Verdampfen der wässrigen Lösung erbält man nur einen Theil in Krystallen, das Meiste zersetzt sich und trocknet zu einer braunen, amorphen, in Wasser leicht löslichen, porösen Masse ein.

Diese Untersuchungen werden fortgesetzt.

Greif 8 wald, den 7. August 1885 .

435. A. W. Hofmann: Ueber die sulfocyanureâure.

[Aus dem Berl. Univ.-Laborat. I. No. DLXXXXIX; vorgetragen in der Sitzung rom Verfasser.]

(Eingegangen am 22. Juli.)

Der leichte Uebergang der normalen Cyansüureäther in Cyanursäureäther, welcher sich, wie 0.01 shausen $^{1}$ ) und ich gezeigt haben, schon bei gewöhnlicher Temperatur vollzieht, hat mich sehon vor einigen Jahren veranlasst, zu versuchen, ob sich nicht auch die Sulfocyansäureäther in ăhnlicher Weise umzubilden vermöchten, und es ist mir in der That gelungen, den Sulfocyansäuremethyläther durch mehrstündiges Erhitzen auf $180-185^{\circ}$ in einen Sulfocyanursäuremethyläther überzuführen. ${ }^{2}$ )

1) Olshausen und Hofmann, diese Berichte III, 269.

2) Hofmann, diese Berichte XII, 1349. 
Das gleichzeitige Auftreten von erheblichen Mengen von Methylsenföl in dieser Reaction liess es zunächst zweifelhaft erscheinen, ob der entstandene Aether die dem $\dot{W}$ ürtz'schen Isocyanursäuremethylather oder aber dem von Olshausen und $\mathrm{mir}^{1}$ ) beschriebenen normalen Cyanursãuremethyläther entsprechende Schwefelverbindung sei. Der Versuch ergab aber alsbald unzweideutig, dass hier der geschwefelte normale, nicht der Isocyanursäureăther vorlag, denn die Einwirkung von Wasser (Salzsäure) bei erhöhter Temperatur lieferte keine Spar von Methylamin und Sulfokohlenoxyd (oder dessen Zersetzungsproducte Schwefelwasserstoff und Kohlensäure), sondern unter stürmischer Entwickelung von Methylmercaptan die gewöhnliche Cyanursäure. Mit dieser Auffassung des neuen Aethers war begreiflich auch der Gedanke gegeben, die bisher unbekannt gebliebene geschwiefelte Cyanursăure zu gewinnen.

Was nun zunăchst die Darstellung des Sulfocyansāuremethyläthers anlangt, Bo kann ich im Allgemeinen auf das bereits früher Mitgetheilte verweisen, darf jedoch eine eigenthümliche, bei dieser Gelegenheit gemachte Beobachtung nicht unerwähnt lassen. Um durch Mangel an Material nicht im Laufe der Arbeit gestört zu werden, hatte ich Hrn. Dr. Bannow gebeten, mir eine grössere Menge Methylsulfocyanat darstellen zu lassen. Das mir schon nach kurzer Frist übersendete Präparat siedete constant bei. $132^{\circ}$ und erwies sich von der vorzüglichen Reinheit, welche die aus den Kahlbaum'schen Werkstätten hervorgehenden Substanzen auszuzeichnen pflegt. Aber dieses reine Schwefelcyanmethyl liess sich zu meinem grossen Erstaunen nicht in die polymere Sulfocyanursäureverbindung überführen. Indem man genan das früher beobachtete Verfahren und zumal die angegebenen Temperaturgrenzen einhielt, ja selbst als man stärker erhitzte, konnten höchstens Spuren der früher so leicht darstellbaren Materie gewonnen werden. Das Schwefelcyanmethyl hatte sich allerdings verändert; es war zum grossen Theil in Methylsenfōl übergegangen, welches sich in der That nạch ein- oder zweimaliger Rectification in Krystallen aus der Flüssigkeit ausschied, so dass dieser Process vielleicht für die Gewinnung von Methylsenföl verwerthbar ist. Das reine Schwefelcyanmethyl polymerisirt sich also nicht; es erlangt aber diese Fähigkeit augenblicklich, wenn man beim Beschicken der Digestionsröhren dem Aether einige Tropfen Salzsăure oder Schwefelsäure zufügt. Offenbar hatte man bei den früheren Versuchen einen nicht ganz reinen Aether verwendet.

Zur Reinigung wurde das rohe Digestionsproduct zunächst wie früher nach dem Auswaschen mit Alkohol ans Eisessig umkrystallisirt. Spăter fand man es zweckmăssiger, das ausgewaschene und getrocknete

I) A. a. 0 . 
Rohproduct alsbald im luftverdünnten Raume zu destilliren. Das Destillat, mit Alkohol gewaschen, ist für die meisten $\mathbf{Z w e c k e}$ rein genug. Auf diese Weise wurden bis zu 50 pCt. der theoretischen Ausbeute erhalten. In ganz besonders reinen und schönen Krystallen gewinnt man die Verbindung, wenn man sie mit Alkohol im Einschlussrohr auf $120^{\circ}$ erhitzt und das Rohr langsam erkalten lässt.

Die Eigenschaften des sulfocyanursauren Methylăthers anlangend will ich noch bemerken, dass er sich obne Veränderung mit Wasser unter Druck auf $180^{\circ}$ erhitzen lässt; bei $220^{\circ}$ tritt ein schwacher Mercaptangeruch auf. Mit concentrirter Salzsäure spaltet er sich schon bei $100^{\circ}$ vollständig in Methylmercaptan und Cyanursäure.

Concentrirte Salpetersäure zerlegt den Aether vollständig; unter Abscheidung von Cyansäure bildet sich dieselbe Methylsulfonsäure, welche J. S. Muspratt ${ }^{1}$ ) durch Behandlung von Sulfocyanmethyl mit Oxydationsmitteln erhalten hat. Das aus so gewonnener Säure dargestellte Baryumsalz gab bei der Analyse 41.58 pCt. Baryum; der Formel

entsprechen $41.89 \mathrm{pCt}$.

$$
\left(\mathrm{CH}_{3} \mathrm{SO}_{3}\right)_{2} \mathrm{Ba}
$$

Nach den im Vorstehenden verzeichneten neuen Erfahrungen über die Polymerisation des Methylsulfocyanats hat man natïrlich auch versucht, die entsprechende Aethyl- und Amylverbindung in ähnlicher Weise umzubilden. Beide liefern in der That in Gegenwart minimaler Mengen von Säure bei einer Temperatur von $190^{\circ}$ die polymeren Aether. Dieselben sind bochsiedende Flüssigkeiten, welche aber nur in geringer Quantität entstehen. Die Versuche wurden nicht in hinreichendem Masssstabe angestellt, um die gebildeten Producte im reinen Zustande zu gewinnen. Dass hier aber in der That sulfocyanursaure Aether entstanden waren, ergab sich unzweideutig, als man diese Flüssigkeiten, mit Salzsäure eingesehlosien, auf $180^{\circ}$ erhitzte; unter Abspaltung von Cyanursäure wurden Aethyl- und Amylmercaptan in Freiheit gesetzt. Der Sulfocyansäuremethyläther wird unter diesen Umständen in Methylmercaptan, Ammoniak, Kohlensäure und Schwefelwasserstoff zerlegt ${ }^{2}$ ).

\section{Darstellung der Sulfocyanursäure.}

Um die Sulfocyanursäure aus dem Methyläther abzuscheiden, wurde derselbe mit Natriumsulfid gemischt und in geschlossenen Röhren drei bis vier Stunden lang einer Temperatur von etwa $250^{\circ}$ ausgesetzt. Das zu diesem Versuche verwendete Natriumsulfid war zum Theil durch Eindampfen einer Mischung von 1 Volum

1) Muspratt, Lieb. Ann. LXV, 259.

2) Vgl. auch Hofmann, diese Berichte. I, 180. 
Natronlauge mit 1 Volum derselben Lauge, welche mit Schwefelwasserstoff gesăttigt war, zum Theil durch Erhitzen von Natriumhydroxyd in einem Strom von Schwefelwasserstoff dargestellt worden. Da sich das nach beiden Verfahren erzeugte Product gleich brauchbar erwiesen hat, so ist in späteren Darstellungen stets das nach dem ersten Verfahren erhaltene Präparat zur Anwendung gekommeu. Das auf die eine oder die andere Weise gewonnene Sulfid muss, da es äusserst hygroskopisch ist, schnell gepulvert und in geschlossenen Flaschen auf bewahrt werden.

Nach der Gleichung

$$
\left[\mathrm{C}_{\mathrm{i}}^{\prime} \mathrm{SCH}_{3}\right]_{3}+3 \mathrm{Na} \mathrm{S}=\left[\mathrm{C}_{\mathrm{N}}^{-\mathrm{SNa}}\right]_{3}+3 \mathrm{NaSCH}_{3}
$$

sollte man auf $10 \mathrm{Gew} .-T h l e$. Aether etwa 11 Gew.-Thle. Natriumsulfid in Anwendung bringen; da letzteres aber stets durch die Einwirkung der Luft beim Eindampfen schon theilweise oxydirt ist, so hat man bei Ausführung des Versuches auf $10 \mathrm{~g}$ Aether geworhnlich $15 \mathrm{~g}$ Natriumsulfid einwirken lassen. Der Inhalt der Röhren ist scheinbar unverändert, beim Oeffnen derselben wird aber alsbald der Geruch nach Methylmercaptan bemerklich; auch giebt sich die Umbildung durch das Verhalten des Productes zum Wasser zu erkennen, in welchem es bei gut geleiteter Operation nahezu vollständig löslich ist. Hat man nicht lange genug oder nicht hoch genug erhitzt, so können bei der Behandlung mit Wasser erhebliche Mengen unangegriffenen Aethers zurückbleiben. Versetzt man die filtrirte Lösung, welche gewöhnlich eine grünliche Färbung hat, mit einem Ueberschusse von Balzsăure, so entwickeln sich Ströme von Methylmercaptan und Schwefelwasserstoff (letzterer von dem überschüssigen Natriumsulfid herrührend), während sich die Sulfocyanursăure als gelb gefürbtes, kaum krystallinisches Pulver niederschlägt.

Fügt man aber zu der nicht allzuverdünnten Lösung die Salzsturo in kleinen Mengen hiuzu, bis die ursprünglich stark alkalische Reaction nahezu verschwunden ist, so entweicht auch jetzt Methylmercaptan, aber die Flüssigkeit erfüllt sich mit einer Masse schwach gelbgefärbter Krystallnadeln, welche durch Abpressen und mehrfaches Umkrystallisiren aus siedendem Wasser oder aus Alkohol, in dem sie etwas schwieriger löslich sind, rein erhalten werden können.

Primäres Natriumsalz der Sulfocyanursäure. Es wird auf dem angegebenen Wege in grossen, oft wohlausgebildeten, starkglänzenden. Krystallen erhalten. Der Formel

$$
(\mathrm{C} \equiv: \equiv \mathrm{N})_{3} \underset{(\mathrm{SH})_{2}}{\mathrm{SNa}}=\mathrm{C}_{3} \mathrm{H}_{2} \mathrm{NaN}_{3} \mathrm{~S}_{3}
$$

entsprechen folgende Werthe:

Bericbte d. D. chem. Gesellschaft. Jahry. XVIII. 


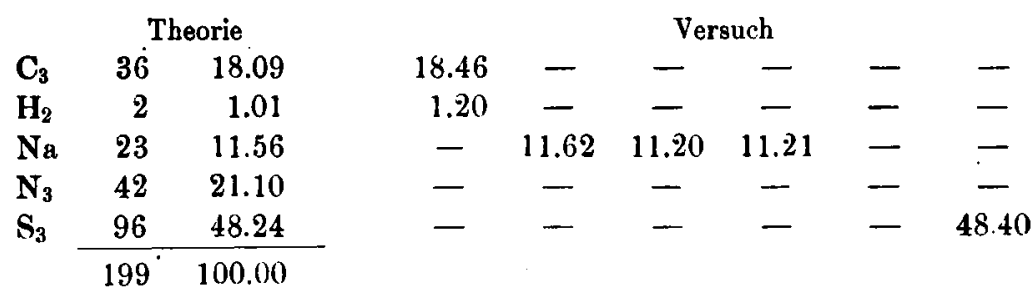

Das sulfocyanursaure Natrium ist selbst in kaltem Wasser leicht löslich; die Lösung hat eine schwach alkalische Reaction und einen intensiv bitteren Geschmack; in Alkohol ist es etwas weniger, aber doch noch immer löslich genug; Aether löst es nicht auf. Es ist begreiflich versucht worden, ob die Sulfocyanursäure im Stande sei, ähnlich wie die Cyanursäure ${ }^{1}$ ), ein schwerlösliches tertiäres Natriumsalz zu bilden. Ein solches Salz ist indessen nicht erhalten worden.

Das trockne sulfocyanursaure Natrium ist in hohem Grade hygroskopisch, eine Eigenschaft, welche die Analyse sehr erschwert. Für letztere ist es bei $140^{\circ}$ getrocknet worden. Die Bestimmung des Schwefels anlangend verdient bemerkt zu werden, dass sie sich nur sehr schwierig durch Schmelzen mit einem Gemisch von Natriumcarbonat und Salpeter, selbst bei Anwendung des von H. Schiff vorgeschlagenen, im Uebrigen so zweckmässigen umgestülpten Tiegels ausführen lässt. Bei einer Reihe von Versuchen wurden erhebliche Verluste erlitten, welche sich erst bei der Analyse nach dem Cariusschen Verfahren vollständig vermeiden liessen. Bei Anwendung dieses Verfahrens werden stets erhebliche Mengen von Cyanursäure gebildet, ein Beweis, wie ausserordentlieh stabil diese Säure ist.

Man kann das Natriumsalz natürlich auch aus der freien Säure darstellen, welche man durch vollständiges Ausfällen der Lösung des Rohproductes mit Salzsäure gewonnen hat. Man muss in diesem Falle Sorge tragen, etwas weniger Natronlauge zu nehmen als zur vollständigen Lösung der Säure bei der Siedetemperatur erforderlich ist.

Nachdem durch Analyse des charakteristiscben Natriumsalzes die Natur der Sulfocyanursäure festgestellt worden war, ist man bestrebt gewesen, die Darstellung dieser Verbindung zu vereinfachen.

Zunächst hat man versucht, die Umsetzung des polymeren Aethers, statt auf trocknem Wege bei hoher Temperatur, in siedender, alkoholischer Lösung zu bewerkstelligen. Der Geruch nach Mercaptan liess in diesem Falle unzweifelhaft eine Reaction erkennen, aber sie erfolgte nur äusserst langsam, und man überzeugte sich bald, dass anf diesem Wege ein Vortheil nicht erzielt wird.

1) Hofmann, diese Berichto III, 769. 
Aussichtsvoller erschien der Gedanke, die Anwendung des Cyanursäuremethyläthers gănzlich zu umgeben. Wenn man sich daran erinnert, dass das Cyanurchlorid mit den Elementen des Wassers leicht in Salzsâure und Cyanursăure übergeht, so liess sich mit einiger Sicherheit erwarten, dass die Sulfocyanursäure auch durch Einwirkung von Schwefelwasserstoff oder Natriumsulfid auf Cyanurchlorid zu gewinnen sein werde.

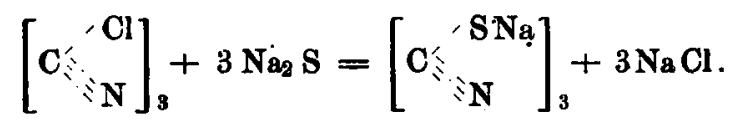

Diese Erwartung hat sich in der That bestätigt. Verreibt man beide Substanzen mit einander, so erfolgt schon bei gewöhnlicher Temperatur eine Wechselwirkung. Gelindes Erwärmen über einem Brenner vollendet die Reaction. Löst man das Product in Wasser, so krystallisirt auf vorsichtigen Zusatz von Salzsäure das oben beschriebene Natriumsalz aus. Durch einen Ueberschuss von Sãure fällt Sulfocyanursăure, welche sich durch Auflösen in Natronlange wieder in das Salz eurück verwandelt. Obwohl die vollständige Uebereinstimmung der Eigenschaften der auf beiden Wegen gewonnenen Substanzen keinen $\mathbf{Z}_{\boldsymbol{w}}$ eifel darüber aufkommen lassen konnte, dass man die Sulfocyanursäure auch aus Cyanurchlorid gewinnen könne, so ist doch auch das mit Hülfe des letzteren dargestellte Natriumsalz analysirt worden. Zwei Versuche gaben 11.49 und 11.58 pCt. Natrinm. Das primăre sulfocyanursaure Natrium enthält, wie bereits oben bemerkt wurde, $11.56 \mathrm{pCt}$. Die Reaction zwischen Cyanurchlorid und Natriumsulfid geht sehr leicht'von Statten, auch ist die Ausbeute eine recht erhebliche. Bedenkt man aber, dass die Darstellung des Cyanurchlorids keineswegs eine leichte, jedenfalls aber eine zeitraubende ist, so muss es dahingestellt bleiben, ob sich die Sulfocyanursäure aus demselben ebenso vortheilhaft, wie aus dem Methylăther gewinnen lässt.

Es sind begreiflich noch andere Wege eingeschlagen worden, um zur Sulfocyanursäure zu gelangen; sie haben aber nicht zum Ziele geführt. Um späteren Bearbeitern dieser Säure Zeit und Mühe zu ersparen, mag dieser vergeblichen Anläufe hier kurz gedacht werden.

Versuche, Kaliumsulfocyanat für sich oder unter Zusatz einiger Tropfen Salzsäure bei wechselnden, bis über $200^{\circ}$ steigenden Temperaturen zu polymerisiren, sind ohne Erfolg geblieben.

Ebenso ist auch die Hoffnung, durch starkes Erhitzen einer Mischung von Kaliảmsulfocyanat mit primärem Kaliumsulfat nach der Gleichung

$$
3(\mathrm{C} \equiv \vdots \equiv \mathrm{NSK})+2 \mathrm{KHSO}_{4}=(\mathrm{C} \equiv \equiv \mathrm{N})_{3} \underset{(\mathrm{SH})_{2}}{\mathrm{SK}}+2 \mathrm{~K}_{2} \mathrm{SO}_{4}
$$

Sulfocyanursäure zu erhalten, nicht in Erfüllung gegangen. Bekanntlich 
entsteht primäres Kaliumcyanurat, wenn Kaliumcyanat mit nascenter Cyansāure zusammentrifft.

Durch Zusammenschmelzen von Persulfocyansäure mit Cyankalium nach der Gleichung:

$$
\mathrm{C}_{2} \mathrm{~N}_{2} \cdot \mathrm{H}_{2} \mathrm{~S}_{3}+\mathrm{KCN}=(\mathrm{C} \equiv \equiv \mathrm{N})_{3} \underset{(\mathrm{SH})_{2}}{\mathrm{SK}}
$$

hat man vergeblich versucht, Sulfocyanursäure zu gewinnen.

Auch Cyanursäure, mit Kaliumsulfid auf $200^{\circ}$ erhitzt, liefert keine Sulfocyanursäure.

Der Gedanke, die Sulfocyanursänre durch die Einwirkung ron Salzsäure auf den Sulfoharnstoff zu gewinnen, liegt, Angesichts der leichten Darstellung der Cyanursäure aus dem Harnstoff par excellence, so nabe, dass dieser Versuch gewiss schon von Vielen angestellt worden ist. Ich habe mich gleichwohl nicht abhalten lassen, diese Reaction nochmals zu studiren. Beim Erhitzen von Sulfoharnstoff in einem Strome trockener Chlorwasserstoffsäure wird keine Sulfocyanursäure geblldet. Es entsteht Salmiak und Sulfocyansäure; welche sich unter Schwefelwasserstoffentwickelung weiter zersetzt.

Schliesslich mag noch eines vergeblichen Versuches gedacht werden, die Sulfocyanursäure ans dem Xanthogenamid zu gewinnen. Durch Einwirkung von Salzsäure auf eine Lösung von Aethylsulfocyanat in Alkohol hat Pinner ${ }^{1}$ ) vor einiger Zeit eine auch früher schon von Salomon ${ }^{2}$ ) auf anderem Wege gewonnene Substanz erhalten, welche er mit dem Namen Isothiurethan bezeichnete. Beim Erhitzen auf $150^{\circ}$ zerfällt das Isothiurethan

$$
\mathrm{CO}<:-\mathrm{S} \mathrm{H}_{2} \mathrm{H}_{5}
$$

geradezu in Mercaptan und Cyanursäure. Es schien von Interesse, das Xanthogenamid

$$
\mathrm{CS}-: \stackrel{-\mathrm{NH}_{2}}{\mathrm{OC}_{2} \mathrm{H}_{5}}
$$

welches sich von der eben genannten Verbindung nur dadurch unterscheidet, dass Schwefel und Sauerstoff die Plätze gewechselt haben, in ähnlicher Richtung zu untersuchen. Das Xanthogenamid hätte sich in Alkohol und Sulfocyanursäure spalten können. Allerdings liegen bereits sehr eingehende Untersuchungen von $\mathrm{Debus}{ }^{3}$ ) über die $\mathrm{Cm}$ wandlungen des Xanthogenamids vor, nach denen sich auch dieser Körper in Cyanursäure und Mercaptan zersetzt. Ich habe diese Versuche gleichwohl noch einmal wiederholt, und namentlich die Einwirkung der Wärme in geschlossenen Apparaten, 'sowie der Salzsäure

1) Pinner, diese Berichte XIV, 1082.

2) Salomon, J. p. Chem. [2] VII, 252.

3) Debus, Lieb. Ann. LXXII, 18. 
auf das Xanthogenamid studirt. Es sind aber genau dieselben Ergebnisse beobachtet worden, welche Debu s beschrieben hat. Sulfocyanursäure konnte auf diesem Wege nicht erhalten werden.

Sulfocyanursäure. Sie wird, wie ich bereits oben bemerkt habe, auf Zusatz eines Ụeberschusses von Salzsăure zu der Lösung des Rohproductes der Einwirkung von Natriumsulfid auf den Methylăther niedergeschlagen. So gewonnen ist sie nicht rein; die kaum krystallinische Säure enthält stets freien Schwefel, wahrscheinlich aus dem dem Natriumsulfid beigemengten unterschwefligsauren Natrium stammend. Seine Gegenwart giebt sich alsbald dadurch zu erkennen, dass sich die Säure nicht vollständig in Ammoniak löst. Rein wird sie gewonnen, wenn man eine siedende verdünnte Lösung des umkrystallisirten Natriumsalzes mit siedender verdünnter Salzsăure zerlegt. Es entsteht ein gelber. Niederschlag, der aus nadelförmigen Krystallen besteht und beim Reiben auffallend elektrisch wird. Die reine Săure lässt sich auch durch Auflösen der Rohsăure in Ammoniak, Trennung des ungelöst bleibenden Sehwefels und Fällung mit Chlorwasserstoffsēure erhalten. Die Operation muss aber mehrfach wiederholt werden, ehe man eine in Ammoniak ohne Trübung lösliche Săure erbãlt.

Die Sulfocyanursäure ist kaum löslich selbst in siedendem Wasser, aber doch noch ausreichend, um demselben eine deutlich saure Reaction zu ertheilen. In Alkohol, Aether, Benzol und selbst Nitrobenżol ist sie ebenfalls nahezu unlöslich. Versuche, sie unter Druck bei $100^{\circ}$. in Alkohol zu lösen, um bei langsamem Erkalten etwas ausgebildetere Krystalle zu erhalten, haben keinen Erfolg erzielt. Die Sulfocyanursăure kann einer Temperatur von $200^{\circ}$ ausgesetzt werden, ohne sich zu verăndern. Beim stärkeren Erhitzen entwickelt sie, den Traditionen der Cyanursăure entsprechend, den Dampf der Sulfocyansāure, wie man leicht durch die Röthung eines mit Eisenchlorid getränkten Papierstreifens erkennt; die grössere Menge Sulfocyanursäure wird aber dabei unter reichlicher Entwickelung von Schwefelwasserstoff verkohlt. Die Sulfocyanursăure wird weder für sich noch in der wässerigen Lösung von Eisenchlorid geröthet. Mit Wasser und selbst mit verdūnnter Salzsäure unter Druck auf $100^{\circ}$ erhitzt, wird die Sulfocyanursäure nur wenig angegriffen; bei $200^{\circ}$ wird sie von Salzsäure vollstăndig in Cyanursäure und Schwefelwasserstoff zerlegt.

Die Sulfocyanursäure entspricht in ihrer Zusammensetzung dem Natriumsalz. Die Formel

erheischt:

$$
\left[\begin{array}{l}
\mathrm{C}_{i \mathrm{~N}}^{-S H} \\
\vdots
\end{array}\right]_{3}=\mathrm{C}_{3} \mathrm{H}_{3} \mathrm{~N}_{3} \mathrm{~S}_{3}
$$


Theorie

\begin{tabular}{lrrrrrr}
$\mathrm{C}_{3}$ & 36 & 20.34 & 21.61 & 20.81 & - & - \\
$\mathrm{H}_{3}$ & 3 & 1.70 & 1.83 & 2.00 & - & - \\
$\mathrm{N}_{3}$ & 42 & 23.73 & - & - & 23.61 & - \\
$\mathrm{S}_{3}$ & 96 & 54.23 & - & - & - & 53.99 \\
\hline & 177 & 100.00 & & & &
\end{tabular}

Bei der ersten Kohlenstoffbestimmung wurde mit einer Mischung von Kupferoxyd und Bleichromat, bei der zweiten ausschliesslich mit Bleichromat verbrannt; trotzdem ist der Kohlenstoff, wie dies bei einer so schwefelhaltigen Substanz nicht auffallen kann, immer noch zu boch gefunden worden.

Die Sulfocyanursäure ist eine kräftige Säure, welche schon in der Kälte die Kohlensäure aus dem Natriumcarbonat austreibt. Sie bildet eine Reihe wohlcharakterisirter Salze, von dẻnen jedoch nur noch das Baryum- und das Silbersalz genauer untersucht worden sind.

Primäres sulfocyanursaures Baryum. Dieses schöne Salz wird leicht erhalten, wenn man die Säure mit siedender Barytlösung behandelt und einen Strom Koblensäure durch die Flüssigkeit leitet. Beim Erkalten der filtrirten Lösung, welche schwach alkalisch geblieben ist, scheiden sich grosse, sehr wohl ausgebildete Prismen aus, welche in auffallender Weise das Licht brechen. Das Salz ist nur mässig löslich in Wasser, unlöslich in Alkohol. Das sulfocyanursaure Baryum krystallisirt mit 2 Mol. Wasser, welche dem Salze in vacuo über Schwefelsäure verbleiben. Da das Wasser, auch bei höherer Temperatur, weit über $200^{\circ}$, in der That selbst bei Temperaturen, bei welchen schon eine leichte Zersetzung eintritt, nicht vollständig entweicht, so ist das vacuumtrockene Salz analysirt worden.

Der Formel

$$
\left[(\mathrm{C} \equiv: \equiv \mathrm{N})_{3}\right]_{2} \underset{(\mathrm{SH})_{4}}{\mathrm{~S}_{2} \mathrm{Ba}}+2 \mathrm{H}_{2} \mathrm{O}=\mathrm{C}_{6} \mathrm{H}_{8} \mathrm{Ba} \mathrm{N}_{6} \mathrm{~S}_{6} \mathrm{O}_{2}
$$

gehören folgende Werthe an:

Theorie

\begin{tabular}{lrr}
$\mathrm{C}_{6}$ & 72 & 13.71 \\
$\mathrm{H}_{8}$ & 8 & 1.52 \\
$\mathrm{Ba}$ & 137 & 26.10 \\
$\mathrm{~N}_{6}$ & 84 & 16.00 \\
$\mathrm{~S}_{6}$ & 192 & 36.57 \\
$\mathrm{O}_{2}$ & 32 & 6.10 \\
\cline { 2 - 3 } & 525 & 100.00.
\end{tabular}

\section{Versuch}

I. II. III. IV.

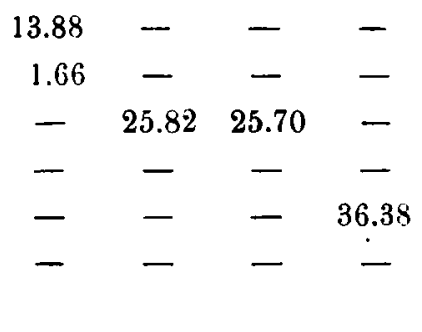


Das Salz entspricht in seiner Zusammensetzung genau dem rọn Wöhler ${ }^{1)}$ analysirten primären Baryumcyanurat mit 2 Mol. Wasser, welche erst bei $280^{\circ}$ entweichen.

Bisweilen setzten sich aus der Lösung, aus welcher der Ueberschuss von Baryt noch nicht durch Kohlensäure entfernt war, grosse, dicke, gelbe Krystalle ab, welche aber nieht willkürlich erzeugt werden konnten. Vielleicht entsprechen sie dem secundären Baryumcyanurat mit $3 \mathrm{Mol}$. Wasser, welches ebenfalls von $\mathrm{Wöhler}^{2}$ ) erforscht worden ist.

Versuche, das Baryumsalz direct aus dem Methyläther durch Einwirkung von (käuflichem) Baryumsulfid bei $280^{\circ} \mathrm{zu}$ erhalten, haben keinen Erfolg gehabt.

Sulfocyanursaures Silber. Versetzt man die Lösung des Natriumsalzes, welche ausgesprochen alkalisch reagirt, mit neutralem Silbernitrat, so entsteht ein canariengelber, amorpher Niederschlag, indem die Flüssigkeit gleichzeitig eine entschieden saure Reaction annimmt. Der Niederschlag muss daher entweder ein secundäres oder ein tertiäres Salz sein. Zuerst an der Luft, dann bei $100^{\circ}$ getrocknet, war der Niederschlag, offenbar in Folge beginnender Zersetzung, missfarbig geworden, daher denn auch bei der Analyse keine übereinstimmenden Zablen erhalten wurden. Wahrscheinlich liegt hier ein secundäres Salz

$$
(\mathrm{C}=. \equiv \mathbf{N})_{\mathbf{3}} \stackrel{(\mathrm{S} \mathrm{Ag})_{\mathbf{8}}}{\mathbf{S H}}
$$

vor, welches $55.24 \mathrm{pCt}$. Silber verlangt; gefunden wurden $56.65,57.6$ und $58.41 \mathrm{pCt}$. Das tertiäre Salz enthält $63.86 \mathrm{pCt}$. Silber.

Fällt man die Lösung des Natriumsalzes mit ammoniakalischer Silberlösung, so entsteht gleichfalls ein gelber Niederschlag, welcher aber schon bei gewöhnlicher Temperatur bald dunkelt und sich beim Kochen schnell schwärzt.

Sulfocyanursaures Blei. Es gleicht dem Silbersalze. Beim Vermischen einer Lösung des alkalischen Natriumsalzes mit neutralem Bleiacetat wird die Flüssigkeit ebenfalls entschieden sauer. Wahrscheinlich hat man es daher auch hier entweder mit einem secundären oder tertiären Salze zu thun.

Sulfocyansaures Kupfer fällt als grünlich-brauner, amorpher Niederschlag beim Vermischen des Natriumsalzes mit Kupfersulfat. Der Niederschlag ist unlöslich in Ammoniak; es wurde vergeblich versucht, eine dem von $W_{\text {öhler }}{ }^{3}$ ) beschriebenen, für die Cyanursäure so charakteristischen violetten Kupferammoniumsalz analoge Verbindung darzustellen.

\footnotetext{
1) Wöhler, Lieb. Ann. LXII, 252.

2) A. a. 0 .

3) A. a. 0 .
} 
Von den übrigen Salzen der Sulfocyanursăure sei bemerkt, dass das Kalium- und Lithiumsalz, erhalten durch Auflösen überschüssiger Säure. in Kalilauge und Lithiumcarbonat, leicht lösliche, schwierig krystallisirbare Salze sind. Das Calcium-, das Strontium- und das Magnesiumbalz, durch Behandlung der Säure mit den Hydraten der genannten Metalle dargestellt, sind lösliche, krystallisirende Verbindungen.

Das Calciumsalz ist von den dreien am wenigsten löslich, aber immer noch viel löslicher als das Baryumsalz; es löst sich auch in Alkohol. Beim langsamen Erkalten einer concentrirten Lösung schiessen wohl ausgebildete, rhombische Tafeln an, die sich zu eigenthümlichen, höchst mannichfaltigen, an maurische Ornamente erinnernden Gruppen vereinigen, wie ich sie bei keinem anderen Salze beobachtet have. Das Strontiumsalz bildet kleine, rhombische Prismen, sehr löslich in Wgsser, auch in Alkohol. Das Magnesiumsalz, ausserordentlich löslich in Wasser, aber unlöslich in Alkohol, wird in nadelförmigen Krystallen erhalten.

Schön krystallisirte Salze entstehen aus dem Natriumsalz durch Zinnchlorid, Eisenchlorid, Manganchlorür, Zinksulfat und Cobaltnitrat.

Das Zinnsalz stellt feine, weisse Nadeln, das Eisensalz feine, zu Gruppen aggregirte, gelbe Nadeln, das Mangansalz dünne, nahezu farblose Krystallblätter, das Zinksalz weisse, das Kobaltsalz endlich schwach röthlich gefärbte, gruppenförmig vereinigte Tafeln dar.

Das Wismuthsalz ist ein gelber, das Nickelsalz ein grünlicber, amorpher Niederschlag; Platin- und Goldsalz stellen rothbraune, amorphe Niederschläge dar.

Hier ist endlich der Ort, auch der Aether der Sulfocyanursäure nochmals zu gedenken. Im Vorhergehenden (S. 2198) wurde bereits erwähnt, dass, ebenso wie der Methyläther, welcher Ausgangspunkt für die vorliegende Arbeit gewesen ist, auch der Aethyl- und Amyläther aus den entsprechenden Sulfocyansäureäthern erhalten werden können. Dieselben lassen sich auch aus dem Natriumsulfocyanurat gewinnen.

Versetzt man eine Lösung dieses Salzes in Alkohol mit etwạs Natronlauge und dann mit Jodmethyl, so scheidet sich nach längerem Stehen schon in der Kälte, fast augenblicklich beim Erwärmen der vielbesprochene Methyläther in kleinen Nadeln von dem oft bestimmten Schmelzpunkte $189^{\circ}$ aus. Werden bei diesem Versuche statt des Jodmethyls die Jodverbindungen des Aethyls und Amyls in Anwendung gebracht, so entstehen, obwohl viel schwieriger, die bereits früher erwähnten Aethyl- und Amylverbindungen.

Noch bequemer erhält man die Sulfocyanursäureäther, wenn man Cyanurchlorid auf die Natriummercaptide der verschiedenen Alkobole und Phenole einwirken lässt. Der Versuch wurde mit den Natriumsalzen der Methyl-, Aethyl- und Amylmercaptane, sowie mit dem Phenylmercaptan in alkoholischer Lösung angestellt. In allen diesen 
Fällen entstehen die zugehörigen Sulfocyanurate, welche indessen nicht weiter untersucht worden sind. Auf das vor einiger Zeit ron mir beschriebene Amidophenylmercaptan 1) wirkt das Cyanurchlorid direct ein, hier werden jedoch die Verhältnisse durch die Gegenwart der Amidogruppe complicirt, so dass die schön krystallisirte Verbindung, welche in dieser Reaction entsteht, besser einer anderen demnächst zu veröffentlichenden Untersuchung über die Einwirkung von Aminen auf den Sulfocyatursauremethylaether und das Cyanurchlorid eingefügt wird.

Am Schlusse dieser Arbeit ist es mir ebenso Bedürfniss wie Pflicht, Hrn. Dr. Otto Borgmann für die thatkräftige und verständnissvolle Hälfe zu danken, welche er mir bei Anstellung der im Vorstehenden beschriebenen Versuche geleistet hat.

436. O. Gubbe: Ueber das optisohe Drehungsvermögen des Invertzuokers.

(Eingegangen am 9. Angust.)

[Vorgetragen in der Sitzung von Hrn. Landolt.]

Die specifische Rotation des Invertzuckers in wässerigen Lösungen wird, wie die aller optisch activen Substanzen abhängig sein:

1) Von dem Wassergehalt der Lösung.

2) Von der Temperatur.

Hierzu kann aber noch ein dritter Einfluss hinzutreten, nămlich derjenige der Säure, welche gedient hatte, um die Invertzuckerlōsung aus Rohrzucker herzustellen und noch in der Flüssigkeit befindlich ist. Reiner zur Abwägung geeigneter Invertzucker lässt sich nicht beschaffen; ebenso bält es schwer, aus der invertirten Rohrzuckerlösung die Säure quantitativ, sowie ohne Aenderung der Invertzuckermenge, zu entfernen, und man ist daher stets auf die Anwendung säurehaltiger Flüssigkeiten angewiesen.

Versuche über die Veränderlichkeit der specifischen Drehung des Invertzuckers mit der Concentration der Lösungen sind bisher nicht angestellt worden. Dagegen liegen Angaben vor über den Einfluss der Temperatur, welcher sich, wie schon Mitscherlich im Jahre 1842

1) Hofmann, diese Berichte XIII, 1223. 